\title{
Micropatterning of Superhydrophobic Silicone Nanofilaments by a Near-Ultraviolet Nd:YAG Laser
}

\author{
Ana Stojanovic, Georg R. J. Artus, and Stefan Seeger ( $₫)$ \\ Institute of Physical Chemistry, University of Zurich, Winterthurerstrasse 190, 8057 Zürich, Switzerland \\ Received: 20 October 2010 / Accepted: 21 October 2010 \\ C The Author(s) 2010. This article is published with open access at Springerlink.com
}

\begin{abstract}
We demonstrate that a recently developed coating composed of superhydrophobic silicone nanofilaments can be selectively functionalized to yield well defined micron-scale patterns of contrasting wettabilities (superhydrophobic/hydrophilic and amphiphobic/amphiphilic). Nanofilament ablation was performed using a near-ultraviolet (UV) laser with a wavelength of $355 \mathrm{~nm}$ and a repetition rate of $10 \mathrm{kHz}$. This is a highly promising approach for open channel microfluidics and microarray analysis due to its simplicity, the chemical and environmental stability of the coating, and the low cost.
\end{abstract}

\section{KEYWORDS}

Superhydrophobic, ultraviolet (UV) laser ablation, wettability, open channels

\section{Introduction}

Artificial superhydrophobic surfaces have generated considerable attention in the last decade due to their significant potential for industrial and scientific applications. Ever since Barthlott and Neinhuis discovered how the chemical and structural nature of the lotus leaf surface provides its strong water repellent properties [1], a variety of materials and techniques have been developed to mimic that effect [2-6]. However patterning extremely hydrophilic and superhydrophobic domains on the same surface has only emerged in recent years and still remains a challenge. Structured surfaces that exhibit lateral patterns of varying wettability can be produced by different techniques, such as microcontact printing [7], photolithography $[8,9]$, deep reactive ion etching [10], plasma etching, as well as by using different chemical methods [11], or combining two or more of the techniques listed

Address correspondence to sseeger $@$ pci.uzh.ch above [12]. Laser-assisted processes are of particular interest to produce devices for microfluidics, bioanalytics, bioreactors, and micro-optics $[13,14]$. Laser micromachining, being a non-contact process, does not lead to problems of mechanical damage and tool wear, and typically yields structures with lateral dimensions on the order of the laser spot diameter. Pattern configuration and channel size can be faithfully reproduced and exactly created according to demand with high geometric flexibility. Also the substrates do not have to be additionally pre- and/or post-treated.

Systems displaying spatially controlled wettability can offer many benefits, such as requiring fewer reagents, faster operations, lower cost, and increased sensitivity. Obvious prerequisites for such lab-on-achip devices are appropriate compartments for the confinement of very small amounts of liquids. These "microcompartments" should have some basic properties: they should have a well defined geometry 
in order to measure the precise amount of liquid contained; they should be able to confine variable amounts of liquid; and they should be accessible in such a way that one can add and extract liquid in a convenient manner.

In the present study, a ultraviolet (UV) laser has been used to create systems with a high wettability contrast. In addition, we have demonstrated the production of lens-shaped droplets composed of two immiscible liquids on an amphiphobic surface and propose possible applications of such systems.

\section{Results and discussion}

Superhydrophobic silicone nanofilaments represent excellent substrates for the production of micron-scale regions with contrasting wettabilities. They can be fabricated using a versatile and inexpensive technique by which a dense layer of silicone nanofilaments is grown onto various substrates $[15,16]$. In this work, optically transparent substrates such as glass and quartz were coated. The combination of the hydrophobicity of the silicone and the surface topography formed by the nanofilaments renders the coated materials superhydrophobic. The resulting coating consists of polymethylsilsequioxane nanofilaments and can be employed in gas phase or solvent phase reactions. It is fully transparent in the visible range and exhibits exceptional chemical and environmental stability $[17,18]$.

Domains of contrasting wettability (e.g., superhydrophobic/hydrophilic and amphiphobic/amphiphilic) were produced by exposing different regions of the substrate to a UV laser causing ablation of the silicone nanofilament coating. Material removal by laser ablation - which is both photochemical and photothermaldepends upon many parameters, such as the laser beam characteristics and ambient conditions. Russo et al. [19] reported that for a nanosecond laser pulse with irradiance less than $10^{8} \mathrm{~W} / \mathrm{cm}^{2}$, the dominant mechanism is thermal vaporization; the temperature of the solid surface increases, and a well defined phase transition occurs, from solid to liquid, liquid to vapor, and vapor to plasma. Photothermal laser ablation of covalently bound coatings such as silane-based monolayers is very effective due to their exceptional thermal, chemical, and photochemical stability [20]. Laser processing usually uses a UV laser to ablate insulating materials due to its small spot size and small heat affect zone (HAZ) [21].

Figure 1(a) shows a representative scanning electron microscope (SEM) image of the structure of the silicone nanofilaments which are irregularly bent and hooked, roughly $10-40 \mathrm{~nm}$ in diameter and range from $50 \mathrm{~nm}$ to a few micrometers in length. An SEM image of a coated quartz substrate with laser ablated parallel lines around $1 \mu \mathrm{m}$ wide and separated by approximately $15 \mu \mathrm{m}$ is shown in Fig. 1(b). In the case of quartz substrates, the lines, and channels are produced by repeating scanning irradiation up to ten times, while in the case of glass substrates shapes are produced by a single scan. This may be explained by the fact that quartz is totally transparent at the wavelength employed, while the glass is highly absorptive. Absorbed energy generates heat, causing the nanofilaments to be removed faster, and a certain amount

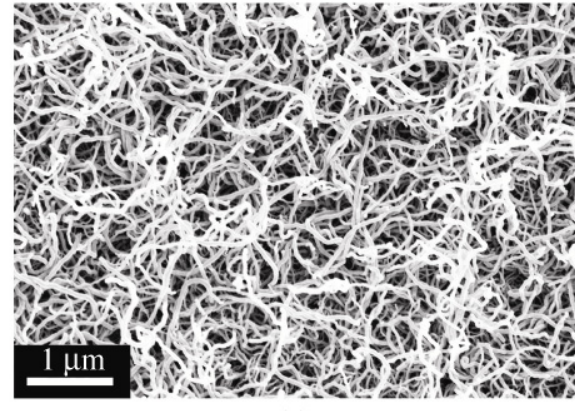

(a)

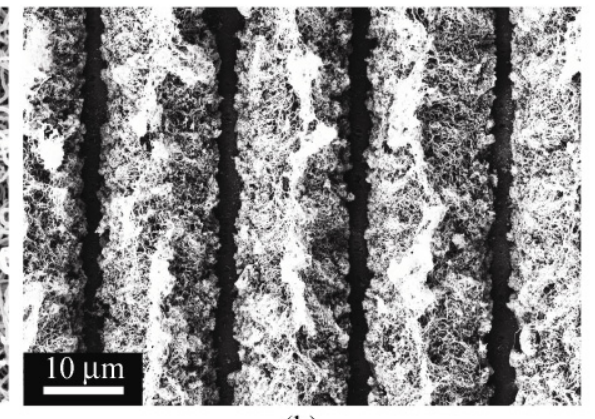

(b)

Figure 1 Scanning electron micrographs: (a) topography of silicone nanofilament coating; (b) laser-ablated lines 1-1.5 $\mu \mathrm{m}$ in width with a separation of approximately $15 \mu \mathrm{m}$ 
of the solidified molten material remains in the cutting kerfs due to the thermal processes associated with nanosecond pulse laser ablation [22, 23].

Using this technique, micron scale hydrophilic structures were created on superhydrophobic silicone nanofilament substrates. Patterned substrates were dipped in an aqueous fluorescent dye solution (the concentration of the dye, fluorescein, was $1 \times 10^{-9} \mathrm{~mol} / \mathrm{L}$ ) for one hour and immediately observed, without rinsing, by inverted fluorescence microscopy. Dye solution applied to the laser-patterned surface spreads only on the ablated hydrophilic regions and does not penetrate the superhydrophobic background.

Figure 2(a) shows the dye absorbed in hydrophilic channels $(20 \mu \mathrm{m}$ wide) on a superhydrophobic background as visualized by an inverted fluorescence microscope, while a representative SEM image of the laser patterned channels is shown in Fig. 2(c).

Photothermal processing with a focused laser beam allows removal of the coating at predefined positions without causing any significant changes to adjacent areas, so is possible to pattern structures such as circles, ellipses or squares. A substrate patterned with various shapes is shown in Fig. 2(b). The hydrophilic domains are visualized using the fluorescent dye as with the channels in Fig. 2(a), and representative SEM images are shown in Figs. 2(d) and 2(e).

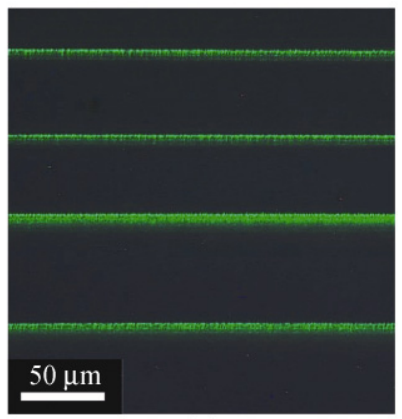

(a)

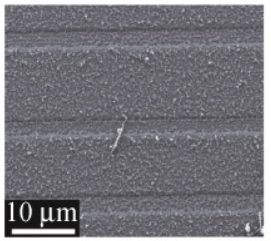

(c)

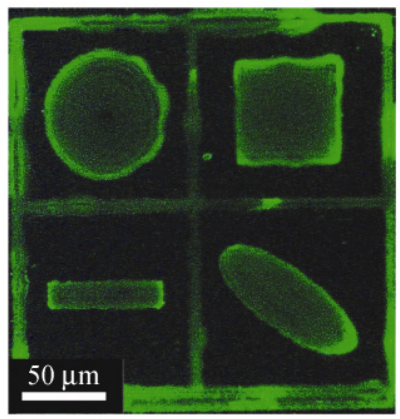

(b)

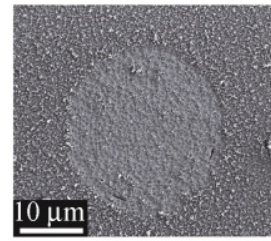

(d)

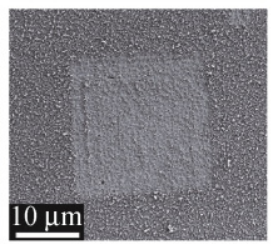

(e)
Figure 2 Production of various hydrophilic patches: (a, b) fluorescent dye solution absorbed on hydrophilic regions (c, d, e) SEM images of various laser-ablated regions
A water droplet on a surface with patterned, superhydrophobic, and completely wetting domains prefers to sit on top of hydrophilic domains. This usually happens even if a droplet is initially pipetted onto a hydrophobic domain, since the droplet dispensed by pipetting always has some kinetic energy and the friction between the surface and the droplet is extremely small [24]. Droplets of aqueous solution remained attached to hydrophilic domains even when the sample was turned upside down. The ability to confine droplets to desired areas on a substrate facilitates their transport and specific analysis. Figure 3 shows colored water droplets confined on irradiated hydrophilic circles (radius $300 \mu \mathrm{m}$ ); in contrast, the sliding angle of the rest of the surface is just $3^{\circ}$. The circles with a radius of $300 \mu \mathrm{m}$ are formed by irradiating concentric circles with a distance of less than $1 \mu \mathrm{m}$ between them. Changing the ablation speed from 2, to 7 to $27 \mu \mathrm{m} / \mathrm{s}$ caused no significant difference in the wetting properties of the ablated substrates (see Fig. S-1 in the Electronic Supplementary Material (ESM)). The contact angle of the irradiated circles was measured by placing $5 \mu \mathrm{L}$ water droplets on irradiated domains and the average value was $57^{\circ}$.

Without further modification, the coating of silicone nanofilaments is ideally non-wetting for water; however, they are almost completely wet by hexadecane and other non-polar solvents. If the nanofilaments were

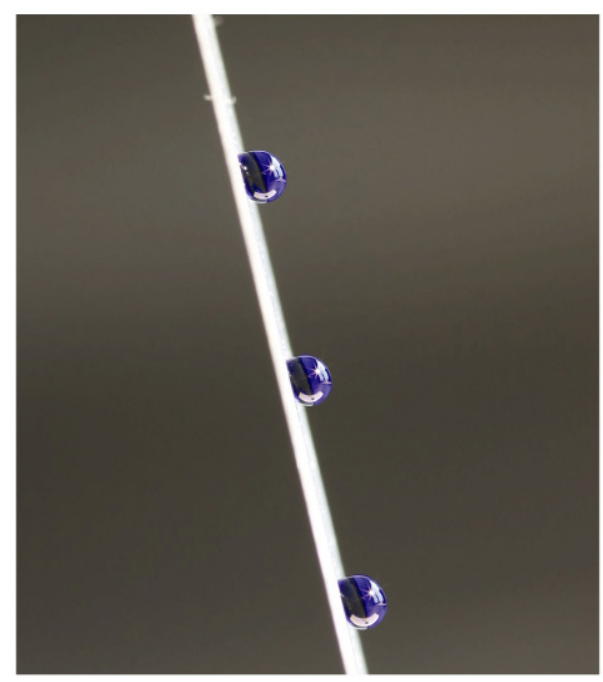

Figure 3 Colored water droplets remain pinned on hydrophilic domains even if the substrate is turned upside-down while the sliding angle of the rest of the surface is just $3^{\circ}$ 
exposed to an oxygen plasma to generate reactive $\mathrm{OH}$ groups and functionalized with a fluorinated species such as $1 \mathrm{H}, 1 \mathrm{H}, 2 \mathrm{H}, 2 \mathrm{H}$-perfluorooctyltrichlorosilane (PFOTS), the coating becomes non-wetting for both polar and non polar liquids although the surface topography remains the same. After treatment with PFOTS, water and hexadecane exhibit contact angles of $168 \pm 2^{\circ}$ and $140 \pm 5^{\circ}$, respectively [25].

Fluorinated samples can be irradiated in a specific pattern to produce features that can take up two different immiscible liquids, and protect aqueous droplets from rapid evaporation, as shown schematically in Fig. 4(a). A water droplet was placed on the central small irradiated circle and oil (hexadecane) was deposited on the surrounding ring-shaped irradiated surface. Upon increasing the volume of the oily liquid using a syringe, the water drop remained trapped inside the oil drop in a defined position, preventing its evaporation. Figure 4(b) shows a colored water droplet inside a

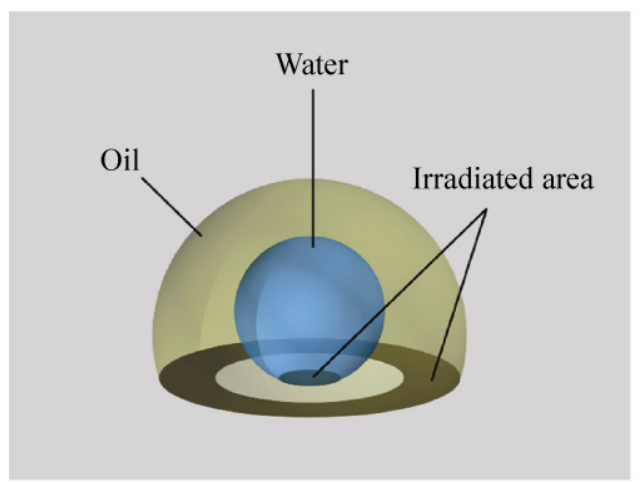

(a)

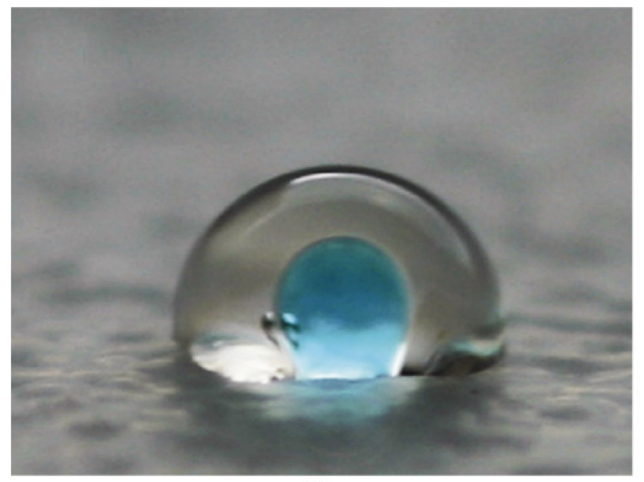

(b)

Figure 4 Systems of two immiscible liquids with suppressed evaporation and accurate positioning of the aqueous liquid: (a) schematic illustration of laser-irradiated patterns; (b) photograph of a small water droplet (dyed with blue ink) contained within hexadecane larger hexadecane droplet. The diameter of the central circle is $200 \mu \mathrm{m}$, the distance between irradiated areas is $300 \mu \mathrm{m}$, and the outer diameter is $1 \mathrm{~mm}$. Of course these values can be varied as required.

By touching the water droplet with a syringe needle is possible to displace it from the surface, forcing the aqueous droplet to float in the "oil chamber" as is shown in Fig. 5.

This opens the possibility of adding and/or extracting aqueous reactants from the oil droplet, which stayed fixed on the irradiated (amphiphilic) domains. Features such as this could be used as "microvessels", where small volumes of aqueous reactants can be kept and analyzed for long periods of time whilst being protected from evaporation. The exceptional chemical and environmental stability of the silicone nanofilaments opens up the possibility of working with a wide variety of polar and non-polar liquids.

\section{Concluding remarks}

We have presented a simple method of patterning onedimensional superhydrophobic silicone nanofilaments on surfaces. The straightforward procedure enables the creation of superhydrophilic/superoleophilic patches of the required shape, with micron precision, embedded into a superhydrophobic or amphiphobic background. The size of the hydrophilic domains can vary from 1.5 microns to a few millimeters, and open channel microfluidic devices are one obvious application. Patterned amphiphilic circles composed of two different liquids provide an interesting platform for storage of volatile liquids and controlled mixing

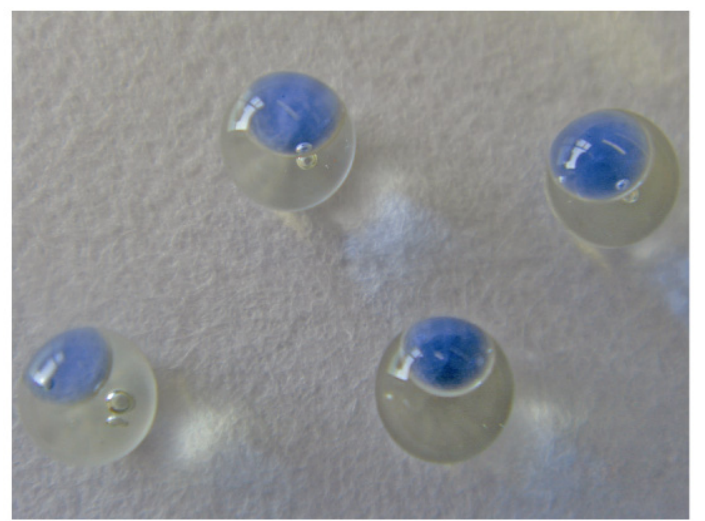

Figure 5 Oil "microvessels" with colored water droplets inside 
studies. Furthermore, ring-shaped features could be used for accurate deposition of aqueous liquids with suppressed evaporation. The fact that the substrates are transparent opens the possibility to investigate confined droplets underneath the substrate by several spectroscopic methods. Different microarrays can be sampled quickly and in large numbers in a parallel mode of operation, enabling the collection of large volumes of data more rapidly than from a single patterned substrate.

\section{Methods}

Glass coverslips $(26 \mathrm{~mm} \times 76 \mathrm{~mm} \times 1 \mathrm{~mm})$ were purchased from Menzel Gläser (Germany). Quartz slides $(26 \mathrm{~mm} \times 76 \mathrm{~mm} \times 0.5 \mathrm{~mm})$ were purchased from SPI Supplies (USA). Prior to coating, an oxygen plasma (Femto, Diener Electronic, Nagold, Germany; 5 min at $100 \mathrm{~W}$ power) was used to generate $\mathrm{OH}$ groups on the substrate surface.

Substrates were coated in a custom built reaction chamber (volume $\sim 700 \mathrm{~mL}$ ) at room temperature. Toluene ( $250 \mathrm{~mL}$, Acros, Extra Dry) was used as the solvent. The water content of the solvent was adjusted inside the reaction chamber by flushing the chamber with dry or humidified nitrogen. A coulometric KarlFisher Titrator DL32 (Mettler Toledo) was used to determine the final water content. Trichloromethylsilane (ABCR, Germany) was introduced into the reaction chamber through a septum with a $\mu \mathrm{L}$ syringe (Hamilton). The reaction mixture was stirred with a remote controlled magnetic stirrer ( $\mathrm{H}+\mathrm{P}$ Labortechnik) at $240 \mathrm{r} / \mathrm{min}$. Substrates were kept in the reaction chamber overnight. Details of the coating procedure can be found in previous publications [16-18].

After coating, the substrates typically exhibit static contact angles of around $160 \pm 2^{\circ}$ and sliding angles of $15 \pm 4^{\circ}$ for $10 \mu \mathrm{L}$ water drops.

To add oleophobic functionality to the coating, the coated samples were first activated in oxygen plasma generator (Femto, Diener Electronics, Nagold, Germany) at $100 \mathrm{~W}$ generator power for $5 \mathrm{~min}$. Functionalization of the activated substrates was subsequently achieved by placing the substrate in a $1 \mathrm{mmol}$ solution of $1 \mathrm{H}, 1 \mathrm{H}, 2 \mathrm{H}, 2 \mathrm{H}$-perfluorooctyltrichlorosilane (PFOTS) in anhydrous toluene (Acros) overnight.
Annealing was performed in a drying oven (Heraeus, Switzerland) at $200{ }^{\circ} \mathrm{C}$ under ambient atmosphere for four hours. After annealing, the contact angle and sliding angle values were improved by roughly $4^{\circ}-6^{\circ}$ and $10^{\circ}-15^{\circ}$, respectively.

Contact angle measurements were performed using a Contact Angle System OCA and associated software (DataPhysics, Germany). For static contact angle measurements, digital drop shape analysis was performed on a $10 \mu \mathrm{L}$ sessile drop of deionised water using the Laplace-Young fitting routine. Sliding angles were measured with the help of a home built tilting table, also on a $10 \mu \mathrm{L}$ drop. Reported values are an average of a minimum of three measurements. All measurements were performed under ambient conditions.

Scanning electron microscopy was performed with a SUPRA 50VP instrument (Zeiss, Germany). Samples were coated with $7 \mathrm{~nm}$ platinum and analyzed at $5 \mathrm{kV}$ using a SE2 detector.

UV laser ablation was performed using a commercial setup for microdissection (mmi CellCut Plus System) consisting of a inverted fluorescence microscope (Nikon, ECLIPSE TE2000-S) with motorized stage, an electronically controlled passive-switched tripled $\mathrm{Nd}$ :YAG laser with a wavelength of $355 \mathrm{~nm}$, with the requisite laser beam delivery and transfer optics. Pulse width was $0.4 \mathrm{~nm}$, frequency $10 \mathrm{kHz}$ and average power $6.6 \mathrm{~mW}$.

\section{Acknowledgements}

We thank the Zentrum für Mikroskopie and Bildanalyse of the University of Zürich for the opportunity to use their facilities and the Swiss National Foundation (SNF) for financial support.

Electronic Supplementary Material: Supplementary material showing lines ablated using different ablation times is available in the online version of this article http://dx.doi.org/10.1007/s12274-010-0062-0 and is accessible free of charge.

Open Access: This article is distributed under the terms of the Creative Commons Attribution Noncommercial License which permits any noncommercial use, distribution, and reproduction in any medium, provided the original author(s) and source are credited. 


\section{References}

[1] Barthlott, W.; Neinhuis, C. The purity of sacred lotus or escape from contamination in biological surfaces. Planta 1997, 202, 1-8.

[2] Callies, M.; Quéré, D. On water repellency. Soft Matter 2005, 1, 55-61.

[3] Feng, X.; Jiang, L. Design and creation of superwetting/ antiwetting surfaces. Adv. Matter. 2006, 18, 3063-3078.

[4] Roach, P.; Shirtclife, N. J.; Newton, M. I. Progress in superhydrophobic surface development. Soft Matter 2008, 4, 224-240.

[5] Zhang, X.; Shi, F.; Niu, J.; Jiang, Y.; Wang, Z. Superhydrophobic surfaces: From structural control to functional application. J. Mater. Chem. 2008, 18, 621-633.

[6] Li, Y.; Zhang, J.; Zhu, S.; Dong, H.; Jia, F.; Wang, Z.; Tang, Y.; Zhang, L.; Zhang, S.; Yang, B. Bioinspired silica surfaces with near-infrared improved transmittance and superhydrophobicity by colloidal lithography. Langmuir 2010, 26, 9842-9847.

[7] Lopez, G. P.; Biebuyck, H. A.; Frisbie, C. D.; Whitesides, G. M.; Imaging of features on surfaces by condensation figures. Science 1993, 260, 647-649.

[8] Kanta, A.; Sedev, R.; Ralston, J. Preparation of silica-ontitania patterns with a wettability contrast. Langmuir 2005, 21, 5790-5794.

[9] Yang, Y. L.; Hsu, C. C.; Chang, T. L. Study on wetting properties of periodical nanopatterns by a combinative technique of photolithography and laser interference lithography. Appl. Surf. Sci. 2010, 256, 3683-3687.

[10] Jokinen, V.; Sainiemi, L.; Franssila, S. Complex droplets on chemically modified silicon nanograss. Adv. Mater. 2008, 20, 3453-3456.

[11] Takachi, M.; Yasuoka, H.; Ohdaira, K.; Shimoda, T.; Matsumura, H. A novel patterning technique using superhydrophobic PTFE thin films by Cat-CVD method. Thin Solid Films 2009, 517, 3622-3624.

[12] Tsougeni, K.; Papageorgiou, D.; Tserepi, A.; Gogolides, E. "Smart" polymeric microfluidics fabricated by plasma processing: Controlled wetting, capillary filling and hydrophobic valving. Lab. Chip 2010, 10, 462-469.

[13] Brandenburg, A.; Edelhäuser, R.; Hutter, F. Integrated optical gas sensors using organically modified silicates as sensitive films. Sensor Actuat. B-Chem. 1993, 11, 361-374.

[14] Pfleging, W.; Torge, M.; Burns, M.; Troullet, V.; Welle, A.; Wilson, S. Laser- and UV-assisted modification of polystyrene surfaces for control of protein adsorption and cell adhesion. Appl. Surf. Sci. 2009, 255, 5453-5457.

[15] Zimmermann, J.; Seeger, S.; Artus, G. R. J.; Jung, S. Superhydrophobic coating. Patent WO/2004113456, June 23, 2004.

[16] Artus, G. R. J.; Jung, S.; Zimmermann, J.; Gautschi, H. P.; Marquardt, K.; Seeger, S. Silicone nanofilaments and their application as superhydrophobic coatings. Adv. Mater. 2008, $18,2758-2762$.

[17] Zimmermann, J.; Reifler, F. A.; Shade, U.; Artus, G. R. J.; Seeger, S. Long term environmental durability of superhydrophobic silicone nanofilament coating. Colloid. Surface. A 2007, 302, 234-240.

[18] Zimmermann, J.; Artus, G. R. J.; Seeger, S. Long term studies on the chemical stability of superhydrophobic silicone nanofilament coating. Appl. Surf. Sci. 2007, 253, 5972-5979.

[19] Russo, R. E.; Mao, X. L.; Yoo, J. H.; Gonzalez, J. J. Laser-induced breakdown spectroscopy. In Laser Ablation. Singh, J. P.; Thakur, S. N., Eds.; Elsevier: USA, 2007; pp 49-82.

[20] Dahlhaus, D.; Franzka, S.; Hasselbrink, E.; Hartman, N. 1D nanofabrication with a micrometer-sized laser spot. Nano Lett. 2006, 6, 2358-2361.

[21] Yavas, O.; Takai, M.; High-speed maskless laser patterning of thin films for giant microelectronics. Jpn. J. Appl. Phys. 1999, 38, 7131-7134.

[22] Dauer, S.; Ehlert, A.; Büttgenbach, S. Rapid prototyping of micromechanical devices using a Q-switched Nd:YAG laser with optional frequency doubling. Sensor Actuat. A-Phys. 1999, 76, 381-385.

[23] Chen, T. C.; Darling, R. B. Laser micromachining of the materials using in microfluidics by high precision pulsed near and mid-ultraviolet Nd:YAG lasers. J. Mater. Process. Tech. 2008, 198, 248-253.

[24] Choi, C. H.; Kim, C. J. Large slip of aqueous liquid flow over a nanoengineered superhydrophobic surface. Phys. Rev. Lett. 2006, 7, 066001-4.

[25] Zimmermann, J.; Rabe, M.; Artus, G. R. J.; Seeger S. Patterned superfunctional surfaces based on a silicone nanofilament coating. Soft Matter 2008, 4, 450-452. 